\title{
AN ANALYTIC METHOD FOR CONVERGENCE ACCELERATION OF CERTAIN HYPERGEOMETRIC SERIES
}

\author{
STANISLAW LEWANOWICZ AND STEFAN PASZKOWSKI
}

\begin{abstract}
A method is presented for convergence acceleration of the generalized hypergeometric series ${ }_{3} F_{2}$ with the argument \pm 1 , using analytic properties of their terms. Iterated transformation of the series is performed analytically, which results in obtaining new fast converging expansions for some special functions and mathematical constants.
\end{abstract}

\section{INTRODUCTION}

We consider convergence acceleration of some generalized hypergeometric series of the type

$$
{ }_{3} F_{2}(a, b, c ; d, e ; \varepsilon) \equiv{ }_{3} F_{2}\left(\begin{array}{c}
a, b, c \\
d, e
\end{array} \mid \varepsilon\right):=\sum_{n=0}^{\infty} \frac{(a)_{n}(b)_{n}(c)_{n}}{n !(d)_{n}(e)_{n}} \varepsilon^{n},
$$

where $\varepsilon= \pm 1$, and $(\alpha)_{n}:=\alpha(\alpha+1) \cdots(\alpha+n-1)$ is Pochhammer's symbol. The function ${ }_{3} F_{2}(a, b, c ; d, e ; 1)$ is one of the fundamental functions of applied mathematics. For special values of its parameters this function can be represented as a ratio of finite products of Gamma function evaluations. In the most general formulae of this type, due to Dixon, Whipple and Watson, two of the parameters $a, b, c, d, e$ are linear combinations of the others [4, vol. $1, \S 4.4,(5)-(7) ; 8$, vol. $1, \S 3.13,(6)-(8)]$. Wimp [14] has shown that these formulae cannot be generalized to the case where four parameters are not interrelated. (Recently, Zeilberger [16] gave a short proof of Wimp's theorem.) As for the case of $\varepsilon=-1$, the most general results are: Kummer's formula for ${ }_{2} F_{1}(a, b ; 1+a-b ;-1)([4$, vol. $1, \S 2.8,(47)])$ and Bailey's formula for ${ }_{3} F_{2}\left(a, b, 1+\frac{1}{2} a ; 1+a-b, \frac{1}{2} a ;-1\right)$ (cf. [4, vol. $\left.\left.1, \S 4.5,(4)\right]\right)$.

Let us recall that many elementary functions $(\tan , \tanh$, cot, coth, csc, $\left.\csc ^{2}, \ldots\right)$, as well as some special functions $\left(\psi, \psi^{\prime}\right.$, Beta,$\left.\ldots\right)$ and mathematical constants $\left(\pi, \pi^{2}\right.$, Catalan's constant $\mathbf{G}$, the lemniscate constant $A$, $\log 2, \ldots)$ can be expressed in terms of the series of type (1.1).

For almost any set of the parameters the series (1.1) is an unsatisfactory way of computing ${ }_{3} F_{2}(\varepsilon)$. Wimp [15, $\left.\S 9.1\right]$ (see also [6]) has proposed an algorithm

Received by the editor December 30, 1992 and, in revised form, August 20, 1993 and March 14, 1994.

1991 Mathematics Subject Classification. Primary 33C20, 65B10; Secondary 65D20.

Key words and phrases. Analytic convergence acceleration, generalized hypergeometric series, polygamma functions, Beta function. 
for computing ${ }_{3} F_{2}(a, b, c ; d, e ; 1)$ numerically, which is based on a threeterm recurrence relation for ${ }_{3} F_{2}(a, b, c ; d+m, e ; 1)(m=0,1, \ldots)$. In this paper we propose an analytic method for the acceleration of convergence of certain series of type (1.1). The term "analytic" means that

$1^{\circ}$ we use explicitly not only values of partial sums of the series (1.1) but also analytic properties of its terms,

$2^{\circ}$ we obtain an analytic form for the transformed series.

The effect of convergence acceleration is remarkable. The transformed series $\sum d_{n}$ is such that $d_{n+1} / d_{n} \rightarrow \lambda_{\varepsilon}$, where $\lambda_{1}=\frac{1}{9}, \lambda_{-1}=-\frac{1}{8}$.

To give the main idea of the proposed approach, let us consider a general series

$$
\sum_{n=0}^{\infty} \varepsilon^{n} a_{n} \quad(\varepsilon= \pm 1)
$$

with the partial sums

$$
s_{n}^{(0)}:=\sum_{j=0}^{n-1} \varepsilon^{j} a_{j} \quad(n=0,1, \ldots) .
$$

The series (1.2) is identical to

$$
s_{0}^{(0)}+\sum_{n=0}^{\infty} \Delta s_{n}^{(0)}
$$

(throughout the paper the forward difference operator $\Delta$ always acts on the subscript $n$ ).

A vast class of methods of accelerating the convergence of the series (1.2) can be considered as recurrent transformation of the sequence $\left\{s_{n}^{(0)}\right\}$ of partial sums of $(1.2)$ to certain suitably chosen sequences $\left\{s_{n}^{(k)}\right\}(k=1,2, \ldots)$, i.e., transformation of (1.3) to

$$
s_{0}^{(k)}+\sum_{n=0}^{\infty} \Delta s_{n}^{(k)}
$$

according to the formula

$$
s_{n}^{(k)}:=s_{n}^{(k-1)}+C_{n}^{(k-1)} \Delta s_{n}^{(k-1)} \quad(n=0,1, \ldots, k=1,2, \ldots) .
$$

The parameters $C_{n}^{(k-1)}$ should be such that for any $k>0, C_{n}^{(k-1)} \Delta s_{n}^{(k-1)} \rightarrow 0$, when $n \rightarrow \infty$. In this case, all the series (1.4) and the series (1.2) have the same sum which we denote by $s$.

Of course, transforming the series (1.2) only makes sense when the rate of convergence of the sequence $\left\{\Delta s_{n}^{(k)}\right\}$ to 0 grows with $k$. Notice that the exact value of $s$ can be obtained as a result of performing the first transformation $\left\{s_{n}^{(0)}\right\} \Rightarrow\left\{s_{n}^{(1)}\right\}$. Indeed, if we define

$$
C_{n}^{(0)}:=\frac{s-s_{n}^{(0)}}{\Delta s_{n}^{(0)}}=\frac{1}{\varepsilon^{n} a_{n}} \sum_{j=n}^{\infty} \varepsilon^{j} a_{j} \quad(n=0,1, \ldots),
$$

then $s_{n}^{(1)}=s$ for every $n$. A useful closed-form expression for the optimum factors $C_{n}^{(0)}$ can be given for trivial series only. In general, we are looking for 
factors which accelerate the convergence of the series. For this purpose, we may use the following relation implied by (1.6):

$$
\frac{C_{n}^{(0)}-1}{C_{n+1}^{(0)}}=\varepsilon \frac{a_{n+1}}{a_{n}} \quad(n=0,1, \ldots) .
$$

Let us assume that

$$
\frac{a_{n+1}}{a_{n}} \sim 1+\rho_{1} n^{-1}+\rho_{2} n^{-2}+\cdots \quad(n \rightarrow \infty)
$$

(this is the case for the series (1.1)). By virtue of a theorem given by Sidi (see [11, Theorem 6.1], or [12, Theorem 2.1]), $C_{n}^{(0)}$ has an asymptotic expansion of the form

$$
C_{n}^{(0)} \sim n^{\tau} \sum_{k=0}^{\infty} c_{k} n^{-k} \quad(n \rightarrow \infty)
$$

with $\tau=(1+\varepsilon) / 2$.

Methods presented in this paper are based on the following facts:

(i) Coefficients of the asymptotic expansion (1.9) of the optimum accelerating factor $C_{n}^{(0)}$ can be effectively calculated using (1.2) and (1.7).

(ii) With methods of Padé approximation applied to this expansion, good rational approximants to this factor can be effectively constructed. (In the simplest case these approximants are identical to those proposed by Knopp [7, $\S 35 \mathrm{~A},(153)]$.)

(iii) For some series (1.2), similar factors $C_{n}^{(k)}$ for $k>0$ can be found provided the approximate expressions for $C_{n}^{(0)}$ are sufficiently simple. This gives a two-dimensional table $\mathbb{S}$ of approximants $s_{n}^{(k)}$ of the sum of the series.

(iv) To any path in the table $\mathbb{S}$ there corresponds a series $\sum d_{n}$, where $d_{0}, \Delta d_{n}$ are entries lying on the path, and analytic expressions for $d_{n}$ are known. It is possible to find a path for which the corresponding series converges to $s$ significantly faster than any series (1.4).

In this paper we show that the process (i)-(iv) can be actually performed for the series ${ }_{3} F_{2}(1, b, c ; d, e ; 1)$ (see Theorem 2.2 in $\S 2$ ) and ${ }_{3} F_{2}(1, b, c ; d, e ;-1)$ with $b+d=c+e$ (see Theorem 3.2 in $\S 3$ ). Some important particular cases are considered in the numerous corollaries and examples, in $\S 2$ for $\varepsilon=1$, and in $\S 3$ for $\varepsilon=-1$. Obviously, the analytic convergence acceleration based on Theorems 2.2 and 3.2 can compete only in a certain limited class of series with purely numerical methods such as Brezinski's $\Theta$-algorithm, $\varepsilon$-algorithm, or Levin's transforms (see [3, $\S \S 2.3,2.7,2.9]$ ).

As a by-product, some identities for contiguous series (1.1) with $\varepsilon=1$ are obtained (see Theorem 2.3).

\section{SERIES FOR $\varepsilon=1$}

Assuming that $\varepsilon=1$, we consider first how the identity (1.7), i.e.,

$$
C_{n}-1=\frac{a_{n+1}}{a_{n}} C_{n+1}
$$

(for simplicity, we drop the superscript in the symbols $C_{n}^{(0)}, C_{n+1}^{(0)}$ ), can be exploited. A general closed-form expression for $C_{n}$, applicable to any series 
(1.2) for $\varepsilon=1$, remains unknown. In the hypergeometric case (1.1) we have

$$
C_{n}={ }_{4} F_{3}\left(\begin{array}{c|c}
1, n+a, n+b, n+c & 1 \\
n+1, n+d, n+e & 1
\end{array}\right),
$$

which does not seem to suggest any practical way of computing this factor.

Let $C_{n}$ satisfy (2.1), and let (1.8) hold. Then a theorem due to Sidi (see [11, Theorem 6.1], or [12, Theorem 2.1]) applies, and we obtain a Poincaré-type asymptotic expansion

$$
C_{n} \sim \sum_{k=-1}^{\infty} c_{k+1} n^{-k}
$$

The coefficients $c_{k}$ can be computed relatively easily by use of the linear relation (2.1). Thereupon, we can introduce the formal power series

$$
F(x):=\sum_{k=0}^{\infty} c_{k} x^{k}
$$

It is known that Padé approximation often gives good rational approximants to such a series, even if it is divergent. As we have $C_{n}=n F\left(n^{-1}\right)$, any Padé approximant $[l / m]_{F}(x)$ gives an approximate value $C_{n}^{(0)}:=n[l / m]_{F}\left(n^{-1}\right)$ of the exact accelerating factor $C_{n}$. It can be checked that the equation $[l / m]_{F}(x)-$ $F(x)=O\left(x^{l+m+1}\right)$ implies the relation $\Delta s_{n}^{(1)}=\Delta s_{n}^{(0)} O\left(n^{-(l+m+1)}\right)$, showing how much faster the transformed series (1.4) for $k=1$ converges compared with the convergence of the original series (1.3). The most natural are perhaps approximants of the type $[m+1 / m]$, as this is the only case where $C_{n}^{(0)}$ defined above is a rational function with respect to $n$, which has all the coefficients of the numerator and the denominator polynomials different from zero, in general.

The simplest transformation of the series (1.3) is based on using the approximant $[1 / 0]_{F}$ to the auxiliary series $(2.3)$. In this case, we use the following

Lemma 2.1. Let

$$
\begin{aligned}
s_{n}^{(0)} & :=\sum_{j=0}^{n-1} a_{j}, \\
s_{n}^{(1)} & :=s_{n}^{(0)}+(\beta n+\gamma) \Delta s_{n}^{(0)},
\end{aligned}
$$

where

$$
\beta=-\frac{1}{\rho_{1}+1}, \quad \gamma=\frac{\rho_{1}+\rho_{2}}{\left(\rho_{1}\right)_{2}},
$$

and $\rho_{1}, \rho_{2}$ are the coefficients of the expansion (1.8). (We assume that $\rho_{1} \neq$ $0,-1)$. Then

$$
\Delta s_{n}^{(1)}=\Delta s_{n}^{(0)} O\left(n^{-2}\right)
$$

Proof. As

$$
\Delta s_{n}^{(1)}=\Delta s_{n}^{(0)}+[\beta(n+1)+\gamma] \Delta s_{n+1}^{(0)}-(\beta n+\gamma) \Delta s_{n}^{(0)},
$$


we have, in view of (1.8),

$$
\begin{aligned}
\Delta s_{n}^{(1)} & =\Delta s_{n}^{(0)}\left((\beta n+\beta+\gamma) \frac{\Delta s_{n+1}^{(0)}}{\Delta s_{n}^{(0)}}-\beta n-\gamma+1\right) \\
& \sim \Delta s_{n}^{(0)}\left[(\beta n+\beta+\gamma)\left(1+\rho_{1} n^{-1}+\rho_{2} n^{-2}+\cdots\right)-\beta n-\gamma+1\right] .
\end{aligned}
$$

For $\beta, \gamma$ given in (2.5) the expression in brackets is equal to $\delta_{2} n^{-2}+\delta_{3} n^{-3}+$ $\cdots=O\left(n^{-2}\right)$.

Remark 1. Knopp [7, §35A, (153)] observed that for $a_{n}=1 /(n+1)^{2}$ it is useful to apply the Kummer transformation, i.e., to replace (1.2) (with $\varepsilon=1$ ) by the series

$$
q b+\sum_{n=0}^{\infty}\left(1-q \frac{b_{n}}{a_{n}}\right) a_{n} \quad\left(b:=\sum_{n=0}^{\infty} b_{n}, \quad q:=\lim _{n \rightarrow \infty} \frac{a_{n}}{b_{n}}\right),
$$

taking $b_{n}:=(n+y) a_{n}-(n+y+1) a_{n+1}$ with a suitably chosen $y$. The above lemma generalizes this observation in a substantial way.

Remark 2. In general, the transformation (2.4) may be iterated. Indeed,

$$
\begin{aligned}
\frac{\Delta s_{n+1}^{(1)}}{\Delta s_{n}^{(1)}} & \sim\left(1+\rho_{1} n^{-1}+\rho_{2} n^{-2}+\cdots\right) \frac{\delta_{2}(n+1)^{-2}+\delta_{3}(n+1)^{-3}+\cdots}{\delta_{2} n^{-2}+\delta_{3} n^{-3}+\cdots} \\
& \sim 1+\bar{\rho}_{1} n^{-1}+\rho_{2} n^{-2}+\cdots .
\end{aligned}
$$

Now, the sequence $\left\{s_{n}^{(2)}\right\}$ can be constructed in an analogous way, provided $\bar{\rho}_{1} \neq 0,-1$.

The following theorem shows that the transformation (2.4) can be effectively iterated for a certain four-parameter family of hypergeometric series.

Theorem 2.2. The series

$$
{ }_{3} F_{2}\left(\begin{array}{c}
1, b, c \\
d, e
\end{array} \mid 1\right)=\sum_{n=0}^{\infty} \frac{(b)_{n}(c)_{n}}{(d)_{n}(e)_{n}} \quad(d, e \neq 0,-1,-2, \ldots, \Re(t)>1),
$$

where $t:=d+e-b-c$, can be transformed according to the following formulae applied for $n=0,1, \ldots$ :

$$
\begin{aligned}
& s_{n}^{(0)}:=\sum_{j=0}^{n-1} \frac{(b)_{j}(c)_{j}}{(d)_{j}(e)_{j}}, \\
& s_{n}^{(k)}:=s_{n}^{(k-1)}+\left(\beta_{k} n+\gamma_{k}\right) \Delta s_{n}^{(k-1)} \quad(k=1,2, \ldots),
\end{aligned}
$$

where

$$
\beta_{k}:=\frac{1}{2 k+t-3}, \quad \gamma_{k}:=1+\frac{b+c}{2 k+t-3}+\frac{b c-(k+d-1)(k+e-1)}{(2 k+t-3)_{2}},
$$

$$
\Delta s_{n}^{(k)}=\frac{(d-b)_{k}(d-c)_{k}(e-b)_{k}(e-c)_{k}(b)_{n}(c)_{n}}{(t-1)_{2 k}(d)_{n+k}(e)_{n+k}} \quad(k=0,1, \ldots) .
$$

The quantities $\Delta s_{n}^{(k)}$ are such that $\Delta s_{n}^{(k)}=O\left(n^{-2 k-t}\right) \quad(n \rightarrow \infty)$. 
If $d-e=b-c$, the formula (2.8) simplifies to

$$
s_{n}^{(k)}:=s_{n}^{(k-1)}+\frac{2 n+3 k-c+d+2 e-5}{2(2 k+t-3)} \Delta s_{n}^{(k-1)} .
$$

Proof. Let $k=0$. Formula (2.10) then agrees with (2.7). Assuming that $k>0$, we obtain from (2.8)

$$
\Delta s_{n}^{(k)}=\left[\beta_{k}(n+1)+\gamma_{k}\right] \Delta s_{n+1}^{(k-1)}-\left(\beta_{k} n+\gamma_{k}-1\right) \Delta s_{n}^{(k-1)}=\Delta s_{n}^{(k-1)} g_{n}^{(k-1)},
$$

where

$$
\begin{aligned}
& g_{n}^{(k-1)}:=\left[\beta_{k}(n+1)+\gamma_{k}\right] \sigma_{n}^{(k-1)}-\left(\beta_{k} n+\gamma_{k}-1\right), \\
& \sigma_{n}^{(k-1)}:=\frac{\Delta s_{n+1}^{(k-1)}}{\Delta s_{n}^{(k-1)}}=1+\rho_{1}^{(k-1)} n^{-1}+\rho_{2}^{(k-1)} n^{-2}+\cdots .
\end{aligned}
$$

According to (2.5) (Lemma 2.1), let

$$
\beta_{k}:=-\frac{1}{\rho_{1}^{(k-1)}+1}, \quad \gamma_{k}:=\frac{\rho_{1}^{(k-1)}+\rho_{2}^{(k-1)}}{\left(\rho_{1}^{(k-1)}\right)_{2}} .
$$

Then $g_{n}^{(k-1)}=O\left(n^{-2}\right)$. Note that

$$
\sigma_{n}^{(k)}=\sigma_{n}^{(k-1)} \frac{g_{n+1}^{(k-1)}}{g_{n}^{(k-1)}} .
$$

It can be shown by induction with respect to $k$ that

$$
\sigma_{n}^{(k)}=\frac{(n+b)(n+c)}{(n+k+d)(n+k+e)},
$$

Now, (2.9) follows from (2.12) and (2.13), while (2.10) follows from (2.11), (2.14) and the equation $\Delta s_{n}^{(0)}=(b)_{n}(c)_{n} /\left[(d)_{n}(e)_{n}\right]$. If $d-e=b-c$, then $t=2(e-c), b c-(k+d-1)(k+e-1)=\frac{1}{2}(2 k+t-2)(k+c+d-1)$, and (2.9) implies the last formula given in the theorem for $s_{n}^{(k)}$.

The following remarks should be added:

Remark 1. If at least one of the differences $d-b, d-c, e-b, e-c$ is a negative integer, then all $\Delta s_{n}^{(k)}$ vanish for a certain $k$, so that the number $s_{0}^{(k)}$ is the sum of the series (2.6). Thus, the procedure described in Theorem 2.2 detects some cases where the sum is a rational expression given in terms of the parameters.

Remark 2. Any Saalschützian series ${ }_{3} F_{2}(a, b, c ; d, e ; 1)$, i.e., such that $d+$ $e=a+b+c+1$, reduces to the form (2.6). This result follows from the identity (see $[8$, vol. $1, \S 3.13,(10)])$

$$
{ }_{3} F_{2}\left(\begin{array}{c|c}
a, b, c \\
d, e
\end{array} \mid 1\right)=\frac{\Gamma(d) \Gamma(e) \Gamma(t)}{\Gamma(a) \Gamma(t+b) \Gamma(t+c)}{ }_{3} F_{2}\left(\begin{array}{c}
t, d-a, e-a \\
t+b, t+c
\end{array} \mid 1\right),
$$

where $t:=d+e-a-b-c, \Re(t)>0, \Re(a)>0$. 
Obviously, the same remark applies to the series ${ }_{3} F_{2}(a, b, c ; a+1, e ; 1)$, where $\Re(e-b-c)>0$.

Remark 3. Some series of the type ${ }_{4} F_{3}(1)$ can be transformed to ${ }_{3} F_{2}(1)$ of one of the above forms. In case of the Saalschützian ${ }_{4} F_{3}(1, b, c, d ; 2, f, g ; 1)$ $(f+g=b+c+d)$, it suffices to apply the identity (see $[9, \S 5.2,(15)])$

$$
\begin{aligned}
{ }_{4} F_{3} & \left(\begin{array}{c}
1, b, c, d \\
2, f, g
\end{array} \mid 1\right) \\
& =\frac{(f-1)(g-1)}{(b-1)(c-1)(d-1)}\left[{ }_{3} F_{2}\left(\begin{array}{c}
b-1, c-1, d-1 \\
f-1, g-1
\end{array} \mid 1\right)-1\right] .
\end{aligned}
$$

The series ${ }_{4} F_{3}(a, b, c, d ; e, c+1, d+1 ; 1)$ can be written as $(\operatorname{see}[9, \S 5.2$, (14)])

$$
\begin{aligned}
& { }_{4} F_{3}\left(\begin{array}{c|c}
a, b, c, d & 1 \\
e, c+1, d+1 & 1
\end{array}\right) \\
& \quad=\frac{d(c-e)}{(c-d) e}{ }_{3} F_{2}\left(\begin{array}{c}
a, b, c \\
e+1, c+1
\end{array}\right)-\frac{c(d-e)}{(c-d) e}{ }_{3} F_{2}\left(\begin{array}{c}
a, b, d \\
e+1, d+1
\end{array} \mid 1\right) .
\end{aligned}
$$

Now, it should be emphasized that the transformations of partial sums of the series (2.6), which are given in Theorem 2.2, are based on the use of some identities for hypergeometric functions of unit argument. Indeed, we have

$$
s_{0}^{(k-1)}+\sum_{n=0}^{\infty} \Delta s_{n}^{(k-1)}=s_{0}^{(k)}+\sum_{n=0}^{\infty} \Delta s_{n}^{(k)}
$$

for $k>0$. Hence it follows from (2.8) for $n=0$ that

$$
\frac{1}{\Delta s_{0}^{(k-1)}} \sum_{n=0}^{\infty} \Delta s_{n}^{(k-1)}=\gamma_{k}+\frac{\Delta s_{0}^{(k)}}{\Delta s_{0}^{(k-1)}} \frac{1}{\Delta s_{0}^{(k)}} \sum_{n=0}^{\infty} \Delta s_{n}^{(k)} .
$$

By virtue of (2.9),

$$
\begin{aligned}
& \frac{\Delta s_{n+1}^{(k)}}{\Delta s_{n}^{(k)}}=\frac{(n+b)(n+c)}{(n+k+d)(n+k+e)} \\
& \frac{\Delta s_{0}^{(k)}}{\Delta s_{0}^{(k-1)}}=\frac{(k+d-b-1)(k+d-c-1)(k+e-b-1)(k+e-c-1)}{(2 k+t-3)_{2}(k+d-1)(k+e-1)}
\end{aligned}
$$

thus (2.15) implies the identity

$$
{ }_{3} F_{2}\left(\begin{array}{c|c}
1, b, c \\
k+d-1, k+e-1
\end{array} \mid 1\right)=\gamma_{k}+\omega_{k 3} F_{2}\left(\begin{array}{c}
1, b, c \\
k+d, k+e
\end{array} \mid 1\right)
$$

with

$$
\omega_{k}=\frac{(k+d-b-1)(k+d-c-1)(k+e-b-1)(k+e-c-1)}{(2 k+t-3)_{2}(k+d-1)(k+e-1)} .
$$

The above identity may be obtained also from some relations which hold for contiguous hypergeometric functions. 
Theorem 2.3. The following identities hold:

$$
\begin{aligned}
& w_{3} F_{2}\left(\begin{array}{c|c}
a, b, c & 1 \\
d-1, e-1 & 1
\end{array}\right) \\
& =(d-a)(e-a) z_{3} F_{2}\left(\begin{array}{c|c}
a-1, b, c & 1 \\
d, e & 1
\end{array}\right) \\
& +\{w+a b c(t-1)-z[(d-a)(e-a)-b c]\}_{3} F_{2}\left(\begin{array}{c|c}
a, b, c & 1 \\
d, e & 1
\end{array}\right),
\end{aligned}
$$

where $t:=d+e-a-b-c, w:=(t-2)_{2}(d-1)(e-1), z:=(t-2)(d+e-2)$ $-(d-1)(e-1)+a b+a c+b c$, and

$$
\begin{aligned}
& (d+e-b-c-1)_{2} d_{{ }_{3}} F_{2}\left(\begin{array}{c|c}
1, b, c & 1 \\
d, e & 1
\end{array}\right) \\
& =\operatorname{de}[(d+e-b-c)(d+e-1)-d e+b c] \\
& +(d-b)(d-c)(e-b)(e-c){ }_{3} F_{2}\left(\begin{array}{c}
1, b, c \\
d+1, e+1
\end{array}\right) .
\end{aligned}
$$

Notice that (2.18) is equivalent to (2.16) for $k=1$.

Proof. Let the symbols $F_{d e}, F_{a}$ and $F$ denote the three functions ${ }_{3} F_{2}(1)$ occurring in (2.17). Further, let $F_{+}:={ }_{3} F_{2}(a+1, b+1, c+1 ; d+1, e+1 ; 1)$. The identities

$$
\begin{aligned}
(d-a)(e-a) F_{a} & =[(d-a)(e-a)-b c] F+(t-1) F_{+}, \\
(t-2)(d-1)(e-1) F_{d e} & =[(t-2)(d-1)(e-1)+a b c] F+z F_{+}
\end{aligned}
$$

can be obtained using Bailey's technique of [2]. Eliminating $F_{+}$from this system yields equation (2.17).

Letting $a=1$ in (2.17), which reduces the second function ${ }_{3} F_{2}$ to 1 , and increasing $d$ and $e$ by 1 , we obtain (2.18).

Example 2.4. The lemniscate constant $A$ is defined in $[13,(14.3-7)]$ by the formula

$$
\begin{aligned}
A & :=\int_{0}^{1}\left(1-x^{4}\right)^{-1 / 2} d x=\sum_{n=0}^{\infty} \frac{(2 n-1) ! !}{(4 n+1)(2 n) ! !} \\
& =\frac{\left[\Gamma\left(\frac{1}{4}\right)\right]^{2}}{4 \sqrt{2 \pi}}=1.31102877714605990523
\end{aligned}
$$

(see also $[1,18.14 .7]$, where this name is given to $\tilde{\omega}:=2 A$ ). Also, we have

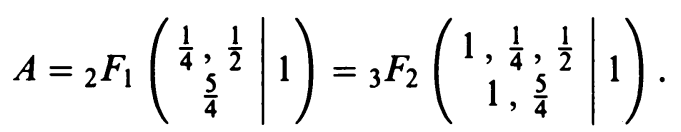

Convergence of this series may be speeded up using Theorem 2.2. As $d-e=$ $b-c$, the recurrence formula $(2.8)$ simplifies to

$$
s_{n}^{(k)}:=s_{n}^{(k-1)}+\frac{2 n+3 k-2}{4 k-3} \Delta s_{n}^{(k-1)} \text {. }
$$


Moreover,

$$
\Delta s_{n}^{(k)}=\frac{\left[\left(\frac{3}{4}\right)_{k}\right]^{2}\left(\frac{1}{2}\right)_{n}}{4(k+1)_{n}\left(k+\frac{1}{2}\right)_{k}\left(n+\frac{1}{4}\right)_{k+1}} .
$$

Transforming the series (1.3) into (1.4) for $k=1,2, \ldots$ yields a twodimensional table $\mathbb{S}$ of numbers $s_{n}^{(k)}$ ( $k$ is a column index, $n$ is a row index). The $r$ th $(r \geq 0)$ antidiagonal of the table, formed by the entries $s_{n}^{(k)}$ with $n+k=r$, depends only on the terms $a_{0}, a_{1}, \ldots, a_{r}$ of the series (1.3) (and on their asymptotic properties). The sum $s$ may be approximated by the partial sums of a fixed series (1.4). It is also possible to use the values $s_{0}^{(k)}$, lying in the first row of $\mathbb{S}$. However, there is a better way of using the table. Indeed, in case of the series (2.19) all the $s_{n}^{(k)}$ are less than its sum and such that $s_{n}^{(k)}<s_{n}^{(k+1)}$ and $s_{n}^{(k)}<s_{n+1}^{(k)}$. Therefore, an optimum sequence of approximations lies on a staircase path in $\mathbb{S}$ along which the approximations grow most quickly. More specifically, we take $s_{n+1}^{(k)}$ as an approximation next to $s_{n}^{(k)}$ if

$$
s_{n+1}^{(k)}-s_{n}^{(k)} \geq s_{n}^{(k+1)}-s_{n}^{(k)}
$$

and $s_{n}^{(k+1)}$ otherwise. Since

$$
s_{n+1}^{(k)}-s_{n}^{(k)}=\Delta s_{n}^{(k)}, \quad s_{n}^{(k+1)}-s_{n}^{(k)}=\frac{2 n+3(k+1)-2}{4(k+1)-3} \Delta s_{n}^{(k)},
$$

the inequality (2.20) holds if and only if $k \geq 2 n$. Thus we obtain the sequence

$$
\begin{array}{llllll}
s_{0}^{(0)} & & & & & \\
s_{1}^{(0)} & s_{1}^{(1)} & s_{1}^{(2)} & & & \\
& & s_{2}^{(2)} & s_{2}^{(3)} & s_{2}^{(4)} & \\
& & & & s_{3}^{(4)} & \ldots
\end{array}
$$

Consequently, (2.19) implies the following series summing to $A$ :

$$
\begin{aligned}
& s_{0}^{(0)}+ \sum_{n=1}^{\infty}\left[\left(s_{n}^{(2 n-2)}-s_{n-1}^{(2 n-2)}\right)+\left(s_{n}^{(2 n-1)}-s_{n}^{(2 n-2)}\right)+\left(s_{n}^{(2 n)}-s_{n}^{(2 n-1)}\right)\right] \\
&= \sum_{n=1}^{\infty}\left(\Delta s_{n-1}^{(2 n-2)}+\frac{8 n-5}{8 n-7} \Delta s_{n}^{(2 n-2)}+\frac{8 n-2}{8 n-3} \Delta s_{n}^{(2 n-1)}\right) \\
&=\sum_{n=1}^{\infty}\left[\frac{\left(\frac{3}{4}\right)_{2 n-2}^{2}\left(\frac{1}{2}\right)_{n-1}}{4(2 n-1)_{n-1}\left(2 n-\frac{3}{2}\right)_{2 n-2}\left(n-\frac{3}{4}\right)_{2 n-1}}\right. \\
&+\frac{\left(\frac{3}{4}\right)_{2 n-2}\left(\frac{3}{4}\right)_{2 n-1}\left(\frac{1}{2}\right)_{n}}{2(2 n-1)_{n}\left(2 n-\frac{3}{2}\right)_{2 n-1}\left(n+\frac{1}{4}\right)_{2 n-1}} \\
&\left.\quad+\frac{\left(\frac{3}{4}\right)_{2 n-2}\left(\frac{3}{4}\right)_{2 n-1}\left(\frac{1}{2}\right)_{n}}{3(8 n-3)(2 n)_{n}\left(2 n-\frac{1}{2}\right)_{2 n-2}\left(n+\frac{1}{4}\right)_{2 n-1}}\right] .
\end{aligned}
$$

It may seem that this series has a complicated form. Notice, however, that it can be written as

$$
A=\sum_{n=1}^{\infty}\left(p_{n}+q_{n}+r_{n}\right)
$$


where $p_{1}:=1$, and

$$
\begin{aligned}
& q_{n}:=\frac{(2 n-1)(4 n-3)(8 n-5)}{2(3 n-2)(8 n-7)(12 n-7)} p_{n}, \\
& r_{n}:=\frac{(2 n-1)(4 n-3)}{3(3 n-1)(8 n-3)} q_{n}, \\
& p_{n+1}:=\frac{8 n-1}{6(12 n+1)} r_{n}
\end{aligned}
$$

for $n=1,2, \ldots$. Note that the quotients $q_{n} / p_{n}, r_{n} / q_{n}, p_{n+1} / r_{n}$ tend to $\frac{1}{9}$ when $n \rightarrow \infty$, so that adding a new term increases the number of correct decimal digits of the approximate sum by approximately $\log _{10} 9=0.95$. Also, it is possible to use the Stirling formula to obtain better estimations of terms (as in Example 2.7).

The above series was summed in Turbo Pascal 5.0 on a microcomputer IBM PC in the extended arithmetic (precision of about 19 decimal digits). Using the terms up to $q_{1}, r_{1}, p_{2}, q_{2}, r_{2}, \ldots, r_{6}$ we obtained approximate values of $A$ with $2.1,3.1,4.0,5.1,6.1,9.0,12.0,14.9,17.9$ correct digits, respectively. This agrees with theoretical estimations and shows that the sum may be computed to almost full machine precision. Notice that the regular analytic form of the series obtained enables one to accelerate its convergence, at least numerically. The same remark applies to other series obtained in this paper.

In $[13, \S 14.4]$, many numerical methods are used for accelerating the convergence of (2.19). The best result is obtained using Levin's $u$-transform, which for 11 terms of the series gives 11.3 digits of its sum. Other methods are much worse, being very sensitive to the influence of rounding errors, even in case where high-precision arithmetic (31-32 decimal digits) is used. We see that using analytic properties of $a_{n+1} / a_{n}$ may increase the speed of convergence and enables us to control the precision of the result.

Example 2.5. If the hypergeometric series (2.6) does not satisfy the condition $|d-e|=|b-c|$, then, according to (2.9), the factor $\beta_{k} n+\gamma_{k}$ in the recurrence formula (2.8) depends on $k$ in a more complex way than, e.g., in the preceding example. However, an optimum path in the table $\mathbb{S}$ may be found also in this case, which is-except perhaps for a finite initial segment-as regular as the one obtained for the lemniscate constant $A$. Such a situation is observed, e.g., for the Beta function defined by

$$
B(p, q):=\int_{0}^{1} x^{p-1}(1-x)^{q-1} d x \quad(\Re(p), \Re(q)>0) .
$$

It is known (see $[5,8.380 .1$ and 8.384.1], [8, vol. $2, \S 13.6,(2)])$ that

$$
B(p, q)=\frac{\Gamma(p) \Gamma(q)}{\Gamma(p+q)}=p^{-1}{ }_{2} F_{1}\left(\begin{array}{c|c}
p, 1-q \\
p+1
\end{array} \mid 1\right)=p^{-1}{ }_{3} F_{2}\left(\begin{array}{c}
1, p, 1-q \\
1, p+1
\end{array} \mid 1\right) .
$$

By virtue of Theorem 2.2, the value of $B(p, q)$ may be computed using the 
formulae

$$
\begin{aligned}
& s_{n}^{(0)}:=\sum_{j=0}^{n-1} \frac{(1-q)_{j}}{j !(j+p)}, \\
& s_{n}^{(k)}:=s_{n}^{(k-1)}+\frac{(2 k+q-1) n+3 k^{2}+(p+2 q-4) k-q+1}{(2 k+q-2)_{2}} \Delta s_{n}^{(k-1)} \quad(k=1,2, \ldots), \\
& \Delta s_{n}^{(k)}=\frac{(1-p)_{k}(p+q)_{k}(1-q)_{n}}{(n+p)_{k+1}(k+q)_{k}(k+1)_{n}} \quad(k=0,1, \ldots) .
\end{aligned}
$$

The optimum path is defined by the following rule: $s_{n}^{(k)}$ should be followed by $s_{n+1}^{(k)}$ if $(k+q)^{2}-p(k+1)>(2 k+q+1) n$, and by $s_{n}^{(k+1)}$ otherwise. This path is very regular for small $p, q$. If, however, we take for instance $p=\frac{3}{4}, q=\frac{9}{2}$, it contains the approximants

$$
\begin{aligned}
& s_{0}^{(0)}, \ldots, s_{4}^{(0)}, s_{4}^{(1)}, s_{4}^{(2)}, s_{5}^{(2)}, s_{5}^{(3)}, s_{5}^{(4)}, s_{6}^{(4)}, s_{6}^{(5)}, s_{6}^{(6)}, s_{7}^{(6)}\left(\text { or } s_{6}^{(7)}\right), \\
& s_{n}^{(2 n-7)}, s_{n}^{(2 n-6)}, s_{n}^{(2 n-5)} \quad(n=7,8, \ldots) .
\end{aligned}
$$

The type of convergence of the final series is the same as in the preceding example.

If the hypergeometric series (2.6) is such that $d=b+1, e=c+1$, then $d-e=b-c$, so that we can apply the last part of Theorem 2.2. Dividing the series by $b c$, we obtain the series

$$
\sum_{n=0}^{\infty} \frac{1}{(n+b)(n+c)} \quad(b, c \neq 0,-1,-2, \ldots) .
$$

Certain mathematical constants as well as many elementary functions (tan, $\tanh$, $\cot$, coth, csc and $\csc ^{2}[1,4.3 .91-93]$ and $\left.[5, \S 1.42]\right)$ and special functions $\left(\psi(z), \psi^{\prime}(z), \Re \psi(i y)\right.$ and $\Im \psi(i y)$; see Example 2.9, or [1, $\S 6.3$ and 6.4]) may be expressed in terms of such series.

Corollary 2.6. The series (2.21) can be transformed according to the following formulae:

$$
\begin{aligned}
& s_{n}^{(0)}:=\sum_{j=0}^{n-1} \frac{1}{(j+b)(j+c)} \\
& s_{n}^{(k)}:=s_{n}^{(k-1)}+\frac{2 n+3 k+b+c-2}{2(2 k-1)} \Delta s_{n}^{(k-1)} \quad(k=1,2, \ldots), \\
& \Delta s_{n}^{(k)}=\frac{k !(b-c+1)_{k}(c-b+1)_{k}}{2^{k}(2 k-1) ! !(n+b)_{k+1}(n+c)_{k+1}} \quad(k=0,1, \ldots),
\end{aligned}
$$

where $n=0,1, \ldots ;$ this implies that $\Delta s_{n}^{(k)}=O\left(n^{-2 k-2}\right) \quad(n \rightarrow \infty)$.

A simplified version of equation (2.23) for $b=c$ was obtained already by Knopp [7, (153)], who, however, did not exploit this result. 
Example 2.7. Let us consider the series (2.21) for $b=c=1$, i.e.,

$$
\sum_{n=0}^{\infty} \frac{1}{(n+1)^{2}}=\frac{1}{6} \pi^{2} \text {. }
$$

We should take

$$
\begin{array}{ll}
s_{n}^{(k)}:=s_{n}^{(k-1)}+\frac{2 n+3 k}{2(2 k-1)} \Delta s_{n}^{(k-1)} & (k=1,2, \ldots), \\
\Delta s_{n}^{(k)}=\frac{(k !)^{3}}{2^{k}(2 k-1) ! !\left[(n+1)_{k+1}\right]^{2}} & (k=0,1, \ldots) .
\end{array}
$$

The optimum path is defined in such a way that $s_{n}^{(k)}$ is followed by $s_{n+1}^{(k)}$ provided $k>2 n+1$, and by $s_{n}^{(k+1)}$ otherwise:

$$
\begin{array}{lllllll}
s_{0}^{(0)} & s_{0}^{(1)} & s_{0}^{(2)} & & & & \\
& & s_{1}^{(2)} & s_{1}^{(3)} & s_{1}^{(4)} & & \\
& & & & s_{2}^{(4)} & s_{2}^{(5)} & s_{2}^{(6)}
\end{array}
$$

Therefore, (2.24) implies the expansion

$$
\begin{aligned}
s_{0}^{(0)} & +\sum_{n=1}^{\infty}\left[\left(s_{n-1}^{(2 n-1)}-s_{n-1}^{(2 n-2)}\right)+\left(s_{n-1}^{(2 n)}-s_{n-1}^{(2 n-1)}\right)+\left(s_{n}^{(2 n)}-s_{n-1}^{(2 n)}\right)\right] \\
& =\sum_{n=1}^{\infty}\left(\frac{8 n-5}{8 n-6} \Delta s_{n-1}^{(2 n-2)}+\Delta s_{n-1}^{(2 n-1)}+\Delta s_{n-1}^{(2 n)}\right)
\end{aligned}
$$

and, finally, we have

$$
\begin{aligned}
\frac{1}{6} \pi^{2}=\sum_{n=1}^{\infty}( & \frac{(8 n-5)[(2 n-2) !]^{3}}{2^{2 n-1}(4 n-3) ! !\left[(n)_{2 n-1}\right]^{2}} \\
& \left.\quad+\frac{[(2 n-1) !]^{3}}{2^{2 n-1}(4 n-3) ! !\left[(n)_{2 n}\right]^{2}}+\frac{[(2 n) !]^{3}}{2^{2 n}(4 n-1) ! !\left[(n)_{2 n+1}\right]^{2}}\right) .
\end{aligned}
$$

Let $n$ be fixed. The first term of the expression in parentheses is asymptotically equal to $(4 \sqrt{2} / 27) \pi \sqrt{\pi} 729^{1-n} / \sqrt{n}$, while the second term is asymptotically 9 times less than the first one, and the third 9 times less than the second one. Thus, the series is similar to the one obtained in Example 2.4. It can be checked that restricting ourselves to the terms for $n \leq 5$, we obtain $\pi^{2}$ with a relative precision of 14.8 decimal digits. Notice that the known series (see [7, (156)])

$$
3 \sum_{n=0}^{\infty} \frac{n !}{(n+1)_{n+2}}
$$

corresponds to the first row of the table $\mathbb{S}$. It also converges qualitatively faster than the original series (each term is at least four times smaller than the preceding one), but it is worse than the series obtained in this example.

Example 2.8. The series

$$
\sum_{n=0}^{\infty} \frac{1}{(2 n+1)(2 n+2)}=\log 2
$$


differs by a constant factor from a series of the type (2.21). It follows from (2.22) and (2.23) that

$$
\begin{array}{ll}
s_{n}^{(0)}:=\sum_{j=0}^{n-1} \frac{1}{(2 j+1)(2 j+2)}, & \\
s_{n}^{(k)}:=s_{n}^{(k-1)}+\frac{2 n+3 k-\frac{1}{2}}{2(2 k-1)} \Delta s_{n}^{(k-1)} & (k=1,2, \ldots), \\
\Delta s_{n}^{(k)}=\frac{(2 k+1) !}{4^{2 k+1}\left(n+\frac{1}{2}\right)_{k+1}(n+1)_{k+1}} & (k=0,1, \ldots) .
\end{array}
$$

The best approximants from the antidiagonals of the table $\mathbb{S}$ are $s_{0}^{(0)}, s_{0}^{(1)}$, $s_{1}^{(1)}, s_{1}^{(2)}, s_{1}^{(3)}, s_{2}^{(3)}, s_{2}^{(4)}, s_{2}^{(5)}, \ldots$. The series

$$
\log 2=\frac{11}{16}+\sum_{n=1}^{\infty} \frac{(2 n) !}{16^{n}}\left(\frac{16 n-1}{(4 n-1)_{2 n+2}}+\frac{16 n+5}{4(4 n+1)_{2 n+2}}+\frac{1}{4(4 n+4)_{2 n+1}}\right),
$$

which has such partial sums, behaves similarly as the series obtained in the preceding example. In particular, the approximant $s_{5}^{(10)}$ gives 15 significant decimal digits of $\log 2$.

Example 2.9. The function $\psi(z):=(\log \Gamma(z))^{\prime}$ (called digamma) and its derivative $\psi^{\prime}$ (known as trigamma) have the following expansions in the complex plane (see $[4$, vol. $1, \S 1.7,(3)$ and $\S 1.9,(10)])$ :

$$
\begin{aligned}
& \psi(z)=-\gamma+(z-1) \sum_{k=0}^{\infty} \frac{1}{(k+1)(k+z)}, \\
& \psi^{\prime}(z)=\sum_{k=0}^{\infty} \frac{1}{(k+z)^{2}} \quad(z \neq 0,-1,-2, \ldots) .
\end{aligned}
$$

Notice that both series are of the form (2.21). Reasoning as above, we can show that for $z \in[1,2)$ the optimum transformation gives

$$
\begin{aligned}
\psi(z)= & -\gamma+\sum_{n=0}^{\infty} \frac{n !(z-2 n-1)_{3 n+1}}{2^{2 n+1}(4 n+1) ! !} \\
& \times\left[\frac{8 n+z+2}{(2 n+1)_{n+1}(z+2 n)_{n+1}}+\frac{(8 n+z+5)(2 n+2-z)}{2(4 n+3)(2 n+2)_{n+1}(z+2 n+1)_{n+1}}\right. \\
& \left.\quad+\frac{(2 n-z+2)(2 n-z+3)}{2(4 n+3)(2 n+3)_{n+1}(z+2 n+2)_{n+1}}\right], \\
\psi^{\prime}(z)= & \sum_{n=0}^{\infty} \frac{[(2 n) !]^{3}}{2^{2 n}(4 n+1) ! !\left[(n+z)_{2 n+1}\right]^{2}} \\
& \times\left\{4 n+z+\frac{1}{2}+\frac{(2 n+1)^{3}}{2(4 n+3)(3 n+z+1)^{2}}\left[4 n+z+2+\frac{4(n+1)^{3}}{(3 n+z+2)^{2}}\right]\right\},
\end{aligned}
$$

respectively. In both cases, adding a new term, i.e., passing trough consecutive three elements of the table $\mathbb{S}$, increases the number of correct decimal digits of the sum by $\log _{10} 729=2.86$ at least. This remains true for real and even 
complex $z$ lying outside the interval $[1,2)$, though the optimum path may depend on $z$.

Example 2.10. In (2.22), (2.23), parameters $b, c$ may have complex values. If, in particular, they are complex conjugate numbers, then the resulting formulae contain real quantities only. Let $b=i, c=-i$, which corresponds to the series $\sum 1 /\left(n^{2}+1\right)$ summing to $\frac{1}{2}(\pi+1)+\pi /\left(e^{2 \pi}-1\right)=2.076674047468581$ $[1,6.3 .13]$. Then

$$
\begin{array}{ll}
s_{n}^{(k)}:=s_{n}^{(k-1)}+\frac{2 n+3 k-2}{2(2 k-1)} \Delta s_{n}^{(k-1)} & (k=1,2, \ldots), \\
\Delta s_{n}^{(k)}=\frac{k ! \prod_{j=1}^{k}\left(j^{2}+4\right)}{2^{k}(2 k-1) ! ! \prod_{j=n}^{n+k}\left(j^{2}+1\right)} \quad(k=0,1, \ldots) .
\end{array}
$$

The optimum sequence of approximants $s_{0}^{(0)}, s_{1}^{(0)}, s_{1}^{(1)}, s_{2}^{(1)}, s_{2}^{(2)}, s_{2}^{(3)}, s_{3}^{(3)}$, $s_{3}^{(4)}, s_{3}^{(5)}, \ldots$ is convergent to the sum of the series as quickly as in the previous examples. In particular, $s_{8}^{(14)}$ gives the result 2.07667404746858117 , which has at least 16 correct digits.

Proceeding in an analogous way, one can obtain new expansions of $\operatorname{coth} x$, $\Im \psi(1+i x)$ and some other functions, as, for instance, we have $[1,6.3 .13]$

$$
\Im \psi(1+i x)=-\frac{1}{2 x}+\frac{1}{2} \pi \operatorname{coth} \pi x=x \sum_{n=1}^{\infty} \frac{1}{n^{2}+x^{2}} .
$$

Let us go back to the general case of series of ${ }_{3} F_{2}(1, b, c ; d ; e ; 1)$ discussed in Theorem 2.2. It can be deduced from the forms obtained for $\beta_{k}, \gamma_{k}$ that the optimum path in the table $\mathbb{S}$ contains, putting aside a finite initial segment, the entries

$$
s_{n}^{(2 n+m)}, s_{n}^{(2 n+m+1)}, s_{n}^{(2 n+m+2)} \quad\left(n=n_{\min }, n_{\min }+1, \ldots\right),
$$

where $m, n_{\min }$ are integers depending on $b, c, d, e$. This implies that the sum of the above series can be expressed as a finite sum corresponding to the initial segment of the path plus the sum of the following three series:

$$
\begin{aligned}
& \sum_{n=n_{\min }}^{\infty}\left(\beta_{2 n+m+1} n+\gamma_{2 n+m+1}\right) \Delta s_{n}^{(2 n+m)}, \\
& \sum_{n=n_{\min }}^{\infty}\left(\beta_{2 n+m+2} n+\gamma_{2 n+m+2}\right) \Delta s_{n}^{(2 n+m+1)}, \sum_{n=n_{\min }}^{\infty} \Delta s_{n}^{(2 n+m+2)} .
\end{aligned}
$$

It can be checked that the first two series are of the type ${ }_{13} F_{12}\left(1, \ldots ; \ldots ; \frac{1}{729}\right)$, and the last is of the type ${ }_{11} F_{10}\left(1, \ldots ; \ldots ; \frac{1}{729}\right)$. The parameters of these new series depend on $b, c, d, e, m$. In particular, if $|d-e| \neq|b-c|$, then as a rule, two numerator parameters and two denominator parameters of ${ }_{13} F_{12}$ are nonrational functions of $b, c, d, e, m$. Obviously, the new series can be simplified considerably in particular cases. In general, however, the theorem relates ${ }_{3} F_{2}(1, b, c ; d, e ; 1)$ to the abovementioned more complex series which are faster convergent. 
Concluding the considerations related to the transformation (2.4), we recall that its parameters $\beta, \gamma$ are determined using the asymptotic expansion of the quotient $\Delta s_{n+1}^{(0)} / \Delta s_{n}^{(0)}=a_{n+1} / a_{n}$. These parameters may also be determined numerically so that three consecutive elements $s_{n}^{(1)}, s_{n+1}^{(1)}, s_{n+2}^{(1)}$ ( $n$ fixed) of the sequence $\left\{s_{n}^{(1)}\right\}$ are identical (and equal to an approximation $\tilde{s}$ of the exact sum $s$ ). We then have

$$
\beta j+\gamma=\frac{\tilde{s}-s_{j}^{(0)}}{\Delta s_{j}^{(0)}} \quad(j=n, n+1, n+2) .
$$

Hence,

$$
\Delta^{2}\left(\frac{\tilde{s}-s_{n}^{(0)}}{\Delta s_{n}^{(0)}}\right)=0
$$

Obviously, $\tilde{s}$ depends on $n$, in general; thus we obtain the following transformation of the sequence $\left\{s_{n}^{(0)}\right\}$ :

$$
\tilde{s}_{n}^{(1)}:=\Delta^{2}\left(\frac{s_{n}^{(0)}}{\Delta s_{n}^{(0)}}\right) / \Delta^{2}\left(\frac{1}{\Delta s_{n}^{(0)}}\right) \quad(n=0,1, \ldots) .
$$

It is easy to verify the following:

(i) The above formula is identical to the first step of Brezinski's $\Theta$-algorithm (see, e.g., $[3, \S 2.9]$ and $[13,(10.3-3)]$ ), more specifically, to passing from the sequence $\left\{\boldsymbol{\Theta}_{0}^{(n)}\right\}$, identical to $\left\{s_{n}^{(0)}\right\}$, to $\left\{\boldsymbol{\Theta}_{2}^{(n)}\right\}$. Also, notice that the method defined by this formula is equivalent to the Lubkin W-method [13, §10.3].

(ii) For some series, $\left\{\tilde{s}_{n}^{(1)}\right\}$ is identical to $\left\{s_{n+1}^{(1)}\right\}$. Such a situation occurs in the case (2.19), where $\Delta^{2}\left(1 / \Delta s_{n}^{(0)}\right)=2$ for $n \geq 0$. In general, however, our analytic transformations differ from the $\Theta$-transform (as well as from other known numerical transforms).

\section{SERIES FOR $\varepsilon=-1$}

In this section we consider series of the form

$$
\sum_{n=0}^{\infty}(-1)^{n} a_{n}
$$

We assume that the $a_{n}$ satisfy condition (1.8). Also in this case accelerating factors $C_{n}^{(k)}$ can be found which produce some new methods for convergence acceleration. The point of departure is, as in $\S 2$, equation (1.7), satisfied by the exact factor $C_{n}^{(0)}$, defined by (1.6). Applying Theorem 6.1 of [11] (see also [12, Theorem 2.1]), we obtain an expansion of this factor into a formal series $c_{0}+c_{1} n^{-1}+c_{2} n^{-2}+\cdots$, where $c_{0}=\frac{1}{2}$. Having computed $c_{k}$, we can replace the exact $C_{n}^{(0)}$ by, e.g., $[m / m]\left(n^{-1}\right)$, where $[m / m]$ is a Padé approximant to the formal power series $c_{0}+c_{1} x+c_{2} x^{2}+\cdots$.

In the simplest case, the exact factor $C_{n}^{(0)}$ is replaced by the number $\frac{1}{2}$ (i.e., the Padé approximant [0/0]). This transformation, defined by $s_{n}^{(1)}:=$ $s_{n}^{(0)}+\frac{1}{2} \Delta s_{n}^{(0)}$, is a typical step of the well-known Euler's transformation. More useful is the following transformation, which is based on the use of $[1 / 1]$. 
Lemma 3.1. Let $s_{n}^{(0)}$ be the partial sum of the series (3.1),

$$
s_{n}^{(0)}:=\sum_{j=0}^{n-1}(-1)^{j} a_{j}
$$

and let

$$
s_{n}^{(1)}:=s_{n}^{(0)}+\frac{n+\zeta}{2 n+\eta} \Delta s_{n}^{(0)}
$$

where

$$
\zeta=-\frac{1}{2}-\frac{\rho_{2}}{\rho_{1}} \quad\left(\text { provided } \rho_{1} \neq 0\right), \quad \eta=2 \zeta+\rho_{1}
$$

Then we have $\Delta s_{n}^{(1)}=-\Delta s_{n}^{(0)} O\left(n^{-3}\right)$.

Proof. In view of (1.8), we have

$$
\begin{aligned}
\Delta s_{n}^{(1)} & =\frac{(-1)^{n+1} a_{n}}{2 n+\eta}\left[(n+\zeta+1) \frac{2 n+\eta}{2 n+\eta+2}\left(1+\sum_{i=1}^{\infty} \rho_{i} n^{-i}\right)-n-\eta+\zeta\right] \\
& =\frac{(-1)^{n+1} a_{n}}{2 n+\eta}\left[(n+\zeta+1)\left(1+\sum_{i=1}^{\infty} \tilde{\rho}_{i} n^{-i}\right)-n-\eta+\zeta\right],
\end{aligned}
$$

where $\tilde{\rho}_{1}:=\rho_{1}-1, \tilde{\rho}_{2}:=\rho_{2}-\rho_{1}+\frac{1}{2} \eta+1$. The expression in brackets can be expanded into a series with respect to the powers of $n^{-1}$. For $\zeta, \eta$ given in Lemma 3.1 the coefficients of $n^{0}$ and $n^{-1}$ vanish, hence the result,

Remark. Recall that in $\S 2$, when $\varepsilon=1$, we obtained the formula $\Delta s_{n}^{(1)}=$ $\Delta s_{n}^{(0)} O\left(n^{-2}\right)$ (see Lemma 2.1). Thus, it might be thought that we obtain here a stronger acceleration effect. This is, however, not confirmed in the case when we choose an optimum sequence of approximations.

The next examples show that the parameters $\zeta$ and $\eta$ can be computed effectively also for the further transformations of series, provided the way in which $a_{n}$ depends on $n$ is sufficiently simple. The most general hypergeometric series to which we are able to apply the proposed method is ${ }_{3} F_{2}(1, b, c ; d, e ;-1)$, where $b+d=c+e$. Obviously, formula (3.4), given in the theorem below, is a consequence of Lemma 3.1.

Theorem 3.2. The series

$$
\begin{aligned}
{ }_{3} F_{2}\left(\begin{array}{c}
1, b, c \\
d, e
\end{array} \mid-1\right) & =\sum_{n=0}^{\infty}(-1)^{n} \frac{(b)_{n}(c)_{n}}{(d)_{n}(e)_{n}} \\
& \left(d, e \neq 0,-1,-2 \ldots, \quad b+d=c+e, \quad \Re(d-c)>\frac{1}{2}\right)
\end{aligned}
$$


may be transformed using the following formulae:

$$
\begin{aligned}
&(3.3) s_{n}^{(0)}:= \sum_{j=0}^{n-1}(-1)^{j} \frac{(b)_{j}(c)_{j}}{(d)_{j}(e)_{j}}, \\
& \begin{aligned}
(3.4) s_{n}^{(k)}:= & s_{n}^{(k-1)}+\frac{n+2 k+\frac{1}{2}(2 e-b+d-5)}{2 n+k+b+d-2} \Delta s_{n}^{(k-1)} \quad(k=1,2, \ldots), \\
(3.5) \Delta s_{n}^{(k)}= & (-1)^{n+k}\left(n+\frac{1}{2}(k+b+d-1)\right) \\
& \times \frac{(d-c)_{k}\left(\frac{1}{2}(e-c+1)\right)_{k}\left(\frac{1}{2}(d-b+1)\right)_{k}(b)_{n}(c)_{n}}{(e)_{n+k}(d)_{n+k}\left(n+\frac{1}{2}(b+d-1)\right)_{k+1}} \quad(k=0,1, \ldots) .
\end{aligned}
\end{aligned}
$$

Here $n=0,1, \ldots$.

Proof. If $k=0$, then (3.5) agrees with (3.3). In case $k>0$, it is sufficient to show that (3.5) satisfies the identity

$\Delta s_{n}^{(k)}=\frac{n+2 k+\frac{1}{2}(2 e-b-d-3)}{2 n+k+b+d} \Delta s_{n+1}^{(k-1)}+\frac{n-k+\frac{1}{2}(2 b-e+c+1)}{2 n+k+b+d-2} \Delta s_{n}^{(k+1)}$,

which follows from (3.4).

Remark 1. If $b+d-1=-2 m(m=0,1, \ldots)$, then the denominator of (3.5) contains $(n-m)_{k+1}$, which vanishes for $n \in\{m, m-1, \ldots, \max (0, m-k)\}$. Thus the formula can be applied for $n>m$ only. The same remark applies to (3.4).

Remark 2. The recurrence relations given in Theorem 3.2 imply the following identity:

$$
\begin{aligned}
{ }_{5} F_{4}\left(\begin{array}{c}
1, b, c, \frac{1}{2}(b+d-1), \frac{1}{2}(k+b+d) \\
\left.k+e-1, k+d-1, k+\frac{1}{2}(b+d-1), \frac{1}{2}(k+b+d)-1 \mid-1\right)
\end{array}\right. \\
=\frac{2 k+\frac{1}{2}(3 e+d-5)}{k+b+d-2} \\
-\frac{(k+e-b-1)(k+b+d-1)\left(k+\frac{1}{2}(e-c-1)\right)\left(k+\frac{1}{2}(d-b-1)\right)}{(k+e-1)(k+d-1)(k+b+d-2)\left(k+\frac{1}{2}(b+d-1)\right)} \\
\quad \times{ }_{5} F_{4}\left(\begin{array}{c}
1, b, c, \frac{1}{2}(b+d-1), \frac{1}{2}(k+b+d+1) \\
k+e, k+d, k+\frac{1}{2}(b+d+1), \frac{1}{2}(k+b+d-1)
\end{array} \mid-1\right) .
\end{aligned}
$$

A regular part of the optimum path in the table $\mathbb{S}$, corresponding to the function ${ }_{3} F_{2}(1, b, c ; d, e ;-1)$, contains only the elements

$$
s_{n}^{(n+m)}, s_{n}^{(n+m+1)} \quad\left(n=n_{\min }, n_{\min +1}, \ldots\right),
$$

where $m, n_{\min }$ depend on $b, c, d, e$. Thus, the sum of the above series can be written as a finite sum, corresponding to the irregular (initial) part of the path, plus

$$
\sum_{n=n_{\min }}^{\infty}\left(\beta_{n+m+1} n+\gamma_{n+m+1}\right) \Delta s_{n}^{(n+m)}+\sum_{n=n_{\min }}^{\infty} \Delta s_{n+1}^{(n+m+1)}
$$


Note that both series are of the type ${ }_{8} F_{7}\left(1, \ldots ; \ldots ; \frac{1}{64}\right)$.

Remark 3. If the condition $\Re(d-c)>\frac{1}{2}$ is relaxed, the series (3.2) is divergent. However, if $d-c, \frac{1}{2}(e-c+1)$, or $\frac{1}{2}(d-b+1)$ is a nonnegative integer, then equations (3.4) and (3.5) still hold, and $\Delta s_{n}^{(k)}$ vanishes for sufficiently large values of $k$. Hence, the analytic transformation of the series (3.2) according to Theorem 3.2 may be viewed as a summation method for a divergent series.

Example 3.3. Theorem 3.2 is directly applicable to the series expansion of

$$
\psi(z)+\gamma=\frac{2(z-1)}{z}{ }_{3} F_{2}\left(\begin{array}{c}
1,1,2-z \\
2,1+z
\end{array} \mid-1\right)
$$

(see [10]). For the expansion of the right-hand side of the above equation, we have

$$
\begin{aligned}
& s_{n}^{(k)}:=s_{n}^{(k-1)}+\frac{n+2 k+z-1}{2 n+k+1} \Delta s_{n}^{(k-1)}, \\
& \Delta s_{n}^{(k)}=\frac{(-1)^{k}(2 n+k+2) k !(z-n-1)_{n+k+1}}{(z+k)_{n+1}\left[(n+1)_{k+1}\right]^{2}} .
\end{aligned}
$$

Let us assume that, for example, $z \in(1,2)$. Then, given arbitrary $k, n \geq 0$, the approximants $s_{n}^{(k)}$ and $s_{n}^{(k+1)}$ lie on the opposite sides of the sum; the same property holds for the numbers $s_{n}^{(k)}$ and $s_{n+1}^{(k)}$, which means that the manner, in $\S 2$, for choosing the best approximants from the antidiagonals of table $\mathbb{S}$ should be reversed here: if $\left|s_{n}^{(k+1)}-s_{n}^{(k)}\right|<\left|s_{n+1}^{(k)}-s_{n}^{(k)}\right|$, i.e., $n \geq k+1$, then $s_{n}^{(k)}$ is followed by $s_{n}^{(k+1)}$, otherwise by $s_{n+1}^{(k)}$. This defines the best path: $s_{0}^{(0)}, s_{1}^{(0)}, s_{1}^{(1)}, s_{2}^{(1)}, \ldots$ and the transformed form of $\psi(z)$ :

$$
\begin{aligned}
\psi(z)+\gamma & =\sum_{n=0}^{\infty}\left[\left(s_{n+1}^{(n)}-s_{n}^{(n)}\right)+\left(s_{n+1}^{(n+1)}-s_{n+1}^{(n)}\right)\right] \\
& =\sum_{n=0}^{\infty}\left(\Delta s_{n}^{(n)}+\frac{3 n+z+2}{3 n+4} \Delta s_{n+1}^{(n)}\right)=\sum_{n=0}^{\infty}\left(p_{n}-q_{n}\right),
\end{aligned}
$$

where $p_{0}:=2(z-1) / z$, and

$$
\begin{aligned}
& q_{n}:=\frac{(n-z+2)(3 n+z+2)}{4(3 n+2)(2 n+z+1)} p_{n}, \\
& p_{n+1}:=\frac{(n+1)(3 n+5)(n+z)^{2}}{(2 n+3)^{2}(2 n+z+2)(3 n+z+2)} q_{n} \quad(n=0,1, \ldots) .
\end{aligned}
$$

The speed of convergence of this series results from the obvious relations $q_{n} / p_{n}$ $\rightarrow \frac{1}{8}, p_{n+1} / q_{n} \rightarrow \frac{1}{8}$. Thus, adding a new term to the series increases the number of exact digits of the result by approximately $\log _{10} 8=0.9$. If, for example, $z=\frac{5}{4}$, then the terms up to $p_{4}$ give the approximate value 0.34976213 , where all the digits are correct. More precise asymptotic expressions for $p_{n}, q_{n}$ can also be obtained. It is worthwhile to compare the above series with the results from Example 2.9.

It is obvious that any series ${ }_{2} F_{1}(1, b ; d ;-1)$ may be written in the form (3.2) with $c=e$. Thus, a simple consequence of the Theorem 3.2 is 
Corollary 3.4. The series

(3.6)

$$
{ }_{2} F_{1}\left(\begin{array}{c}
1, b \\
d
\end{array} \mid-1\right)=\sum_{n=0}^{\infty} \frac{(-1)^{n}(b)_{n}}{(d)_{n}} \quad(d \neq 0,-1,-2 \ldots, \quad \Re(d-b)>1)
$$

may be transformed using for $n=0,1, \ldots$ the formulae

$$
\begin{aligned}
& s_{n}^{(0)}:=\sum_{j=0}^{n-1} \frac{(-1)^{j}(b)_{j}}{(d)_{j}} \\
& s_{n}^{(k)}:=s_{n}^{(k-1)}+\frac{n+2 k+d-\frac{5}{2}}{2 n+k+b+d-2} \Delta s_{n}^{(k-1)} \quad(k=1,2, \ldots), \\
& \Delta s_{n}^{(k)}=\frac{(-1)^{n+k}\left(\frac{1}{2}\right)_{k}(2 n+k+b+d-1)(d-b)_{2 k}(b)_{n}}{(d)_{n+k}(2 n+b+d-1)_{2 k+1}} \quad(k=0,1, \ldots) .
\end{aligned}
$$

Remark 1. If $d-b$ is a negative integer, the series (3.6) diverges. However, $\Delta s_{n}^{(k)}$ defined by equation (3.8) vanishes for sufficiently large $k$, if $d-b$ is a negative integer. Thus, the transformation of the series (3.6) according to Corollary 3.4 is a summation method for a divergent hypergeometric series.

Remark 2. It is interesting that, given $b, d$, equation (3.7) may serve to accelerate the convergence of unique hypergeometric series, different from ${ }_{2} F_{1}(1, b ; d ;-1)$, namely, ${ }_{2} F_{1}\left(b+d-1, \frac{1}{2}(b-d)+1 ; \frac{1}{2}(b+3 d)-1 ;-1\right)$. Note, however, that the sum of the latter series can be expressed in terms of the Gamma function [4, vol. $1, \S 2.8,(47)]$.

Remark 3. Under the assumption that $d>b>0$, we obtain the same optimum path as in Example 3.3.

Example 3.5. Corollary 3.4 enables us to obtain a rapidly convergent expansion for $\sqrt{2}$. It is known that

$$
\begin{aligned}
\sqrt{1+x} & ={ }_{1} F_{0}\left(\frac{-\frac{1}{2}}{-} \mid-x\right)=1+\frac{1}{2} x_{2} F_{1}\left(1, \frac{1}{2} \mid-x\right) \\
& =1+\sum_{n=1}^{\infty} \frac{(-1)^{n-1}(2 n-3) ! !}{(2 n) ! !} x^{n}
\end{aligned}
$$

Now, we can set for $x=1$

$$
\begin{aligned}
s_{n}^{(0)} & :=1+\sum_{j=1}^{n-1} \frac{(-1)^{j-1}(2 j-3) ! !}{(2 j) ! !}, \\
s_{n}^{(k)} & :=s_{n}^{(k-1)}+\frac{n+2 k-\frac{3}{2}}{2 n+k-\frac{3}{2}} \Delta s_{n}^{(k-1)} \quad(k=1,2, \ldots), \\
\Delta s_{n}^{(k)} & =\frac{(-1)^{n+k-1} 2^{k}\left(\frac{1}{2}\right)_{k}\left(\frac{3}{2}\right)_{2 k}(2 n-3) ! !\left(2 n+k-\frac{1}{2}\right)}{\left(2 n-\frac{1}{2}\right)_{2 k+1}(2 n+2 k) ! !} \quad(k=0,1, \ldots) .
\end{aligned}
$$

The optimum sequence of approximations of $\sqrt{2}$ is: $s_{1}^{(0)}, s_{2}^{(0)}, s_{2}^{(1)}, s_{3}^{(1)}$, 
$s_{3}^{(2)}, \ldots$. Thus, we obtain the expansion

$$
\sqrt{2}=1+2 \sum_{n=1}^{\infty} \frac{(2 n-3) ! !(4 n-3) ! !}{32^{n}(n-1) !}\left(\frac{12}{\left(2 n-\frac{1}{2}\right)_{2 n-1}}-\frac{6 n-1}{n\left(2 n+\frac{3}{2}\right)_{2 n-1}}\right),
$$

where each term corresponds to a shift in the table $\mathbb{S}$ by two entries, i.e., to two terms of the original series. An asymptotic expression may be found for this term, like in the next example. Let us limit ourselves to the remark that the $n$th term is a difference of two numbers, $g_{n}$ and $h_{n}$, say, such that the quotients $g_{n} / h_{n}$ and $h_{n} / g_{n-1}$ are both asymptotically equal to 8 . Thus, adding a new term to the series increases the number of correct digits of the result approximately by $\log _{10} 64=1.8$. This is confirmed by computations performed for $n \leq 1,2,3,4$, as we obtain $2.6,4.5,6.4,8.3$ decimal digits, respectively.

Example 3.6. Assume now that $d=b+1$, which gives the series

$$
\sum_{n=0}^{\infty} \frac{(-1)^{n}}{n+b}
$$

having the sum $\frac{1}{2}\left[\psi\left(\frac{1}{2}(b+1)\right)-\psi\left(\frac{1}{2} b\right)\right]=\frac{1}{2} G(b)$ [4, vol. 1, §1.8]. Notice that (3.6) reduces in this case to a series which is equal to (3.9) multiplied by $b$. Using the formula (3.8), we should, therefore, divide its right-hand side by $b$. In particular, for $b=\frac{1}{3}$ (the sum of (3.9) is then $\pi / \sqrt{3}+\log 2$; see [5, $0.239 .1]$ ) and $b=\frac{1}{2}$ (the sum is $\pi / 2 ;[5,0.232 .2]$ ) this gives the sequence $s_{0}^{(0)}, s_{1}^{(0)}, s_{1}^{(1)}, s_{2}^{(1)}, \ldots$ corresponding to the series

$$
\sum_{n=1}^{\infty}\left[\left(s_{n}^{(n-1)}-s_{n-1}^{(n-1)}\right)+\left(s_{n}^{(n)}-s_{n}^{(n-1)}\right)\right]=\sum_{n=1}^{\infty}\left(\Delta s_{n-1}^{(n-1)}+\frac{3 n+d-\frac{3}{2}}{3 n+2 d-1} \Delta s_{n}^{(n-1)}\right) .
$$

For $b=\frac{1}{2}$, this implies the expansion

$$
\pi=\sum_{n=1}^{\infty}(n-1) !\left[\left(\frac{1}{2}\right)_{n-1}\right]^{2}\left(\frac{3 n-2}{\left[\left(n-\frac{1}{2}\right)_{n}\right]^{2}(n)_{n-1}}-\frac{3 n-1}{\left[\left(n+\frac{1}{2}\right)_{n}\right]^{2}(n+1)_{n-1}}\right)
$$

Its first three terms give $3.1415878 \ldots$, which is a value of $\pi$ correct to 5.8 decimal digits.

In case $b=\frac{2}{3}$ (sum of $(3.9)$ is $\pi / \sqrt{3}-\log 2$; see [5,0.239.2]) and for $b=1$ (the sum is $\log 2$ ) the optimum sequence is $s_{0}^{(0)}, s_{0}^{(1)}, s_{1}^{(1)}, s_{1}^{(2)}, \ldots$ Hence, we obtain the series

$$
\sum_{n=1}^{\infty}\left[\left(s_{n-1}^{(n)}-s_{n-1}^{(n-1)}\right)+\left(s_{n}^{(n)}-s_{n-1}^{(n)}\right)\right]=\sum_{n=1}^{\infty}\left(\frac{3 n+d-\frac{5}{2}}{3 n+2 d-3} \Delta s_{n-1}^{(n-1)}+\Delta s_{n-1}^{(n)}\right) .
$$

For $b=1$ this gives the expansion

$$
\log 2=3 \sum_{n=1}^{\infty} \frac{[(2 n-2) !]^{2}}{4^{n}(4 n-3) !}\left(1-\frac{2 n-1}{4(4 n-1)}\right) .
$$

It can be verified that the $n$th term of the last series is asymptotically equal to $\frac{1}{16} \sqrt{2 \pi} 64^{1-n} / \sqrt{n}$ (this applies also to the series (3.10)). In particular, the 
approximant $s_{7}^{(8)}$ gives 14.2 decimal digits of $\log 2$. Note that this result is much better than the one obtained in $\S 2$. Indeed, the series, which has been transformed there, is obtained by summing pairs of subsequent terms of (3.9); to obtain 15 correct digits, we needed about 30 terms of (3.9).

Let us consider the series

$$
\begin{aligned}
\sum_{n=0}^{\infty} \frac{(-1)^{n}}{(n+b)(n+c)}=\frac{1}{b c}{ }_{3} F_{2}\left(\begin{array}{c}
1, b, c \\
b+1, c+1
\end{array} \mid-1\right) \\
\quad(b, c \neq 0,-1,-2, \ldots) .
\end{aligned}
$$

Notice that the above ${ }_{3} F_{2}$ is of the form (3.2) with $d=c+1, e=b+1$. Thus, we obtain the following.

Corollary 3.7. The series (3.11) may be transformed using

$$
\begin{aligned}
& s_{n}^{(0)}:=\sum_{j=0}^{n-1} \frac{(-1)^{j}}{(j+b)(j+c)}, \\
& s_{n}^{(k)}:=s_{n}^{(k-1)}+\frac{n+2 k+\frac{1}{2}(b+c)-1}{2 n+k+b+c-1} \Delta s_{n}^{(k-1)} \quad(k=1,2, \ldots), \\
& \Delta s_{n}^{(k)}=\frac{(-1)^{n+k} k !\left[n+\frac{1}{2}(k+b+c)\right]\left[\frac{1}{2}(b-c)+1\right]_{k}\left[\frac{1}{2}(c-b)+1\right]_{k}}{\left[n+\frac{1}{2}(b+c)\right]_{k+1}(n+b)_{k+1}(n+c)_{k+1}} \\
& (k=0,1, \ldots)
\end{aligned}
$$

for $n=0,1, \ldots$. If $b=c$, the last formula simplifies to

$$
\Delta s_{n}^{(k)}=(-1)^{n+k}\left(n+\frac{1}{2} k+b\right)\left(\frac{k !}{(n+b)_{k+1}}\right)^{3} .
$$

For $b \neq c$ the series (3.11) can be written as a combination of series from Example 3.6, i.e., in terms of the $\psi$ function. If $b=c$, its sum is $\frac{1}{4}\left[\psi^{\prime}\left(\frac{1}{2} b\right)-\right.$ $\left.\psi^{\prime}\left(\frac{1}{2}(b+1)\right)\right]$; note that a series expansion for $\psi^{\prime}$ has been obtained in Example 2.9. However, it may be inferred that the optimum (in the sense defined) convergence acceleration of the series (3.11) would give simpler and better results than using the same procedure for each of the two series separately. Indeed, this is the case.

Example 3.8. If $b=c=1$, the series (3.11) has the sum $\frac{1}{12} \pi^{2}$. The optimum sequence $s_{0}^{(0)}, s_{1}^{(0)}, s_{1}^{(1)}, s_{2}^{(1)}, \ldots$ of approximants yields the series

$$
\begin{aligned}
\sum_{n=1}^{\infty}\left[\left(s_{n}^{(n-1)}\right.\right. & \left.\left.-s_{n-1}^{(n-1)}\right)+\left(s_{n}^{(n)}-s_{n}^{(n-1)}\right)\right] \\
& =\frac{1}{2} \sum_{n=1}^{\infty}\left[(3 n-1)\left(\frac{(n-1) !}{(n)_{n}}\right)^{3}-3 n\left(\frac{(n-1) !}{(n+1)_{n}}\right)^{3}\right] \\
& =\frac{1}{2} \sum_{n=1}^{\infty}(21 n-8)\left(\frac{(n-1) !}{(n+1)_{n}}\right)^{3} .
\end{aligned}
$$


The $n$th term of the last series is asymptotically equal to $21 \pi \sqrt{\pi} 64^{-n} / \sqrt{n}$, i.e., the convergence acceleration has the same character as in the preceding example.

If $b=c=\frac{1}{2}$, the series (3.11) has the sum $4 \mathbf{G}$, where $\mathbf{G}$ is the so-called Catalan's constant, approximately equal to 0.915965594177219015 (see, e.g., $[8$, vol. 2, p.293]). Proceeding as before, we obtain the expansion

$$
\mathbf{G}=1-\frac{1}{8} \sum_{n=1}^{\infty}\left[3 n-\frac{1}{2}-8(3 n+1)\left(\frac{n}{4 n+1}\right)^{3}\right]\left(\frac{(n-1) !}{\left(n+\frac{1}{2}\right)_{n}}\right)^{3}
$$

As before, adding one term to a partial sum of this expansion, i.e., adding two terms of the original series, increases the accuracy of the result by $\log _{10} 64=1.8$ decimal digits at least.

The parameters $b, c$ may have complex values. In case of $\sum(-1)^{n} /$ $\left(n^{2}+n+1\right)$ we have $b+c=b c=1$, hence

$$
\begin{aligned}
& s_{n}^{(k)}:=s_{n}^{(k-1)}+\frac{n+2 k-\frac{1}{2}}{2 n+k} \Delta s_{n}^{(k-1)} \quad(k=1,2, \ldots), \\
& \Delta s_{n}^{(k)}=\frac{(-1)^{n+k} k !\left(n+\frac{1}{2}(k+1)\right) \prod_{h=1}^{k}\left(h^{2}+\frac{3}{4}\right)}{\left(n+\frac{1}{2}\right)_{k+1} \prod_{h=n}^{n+k}\left(h^{2}+h+1\right)} \quad(k=0,1, \ldots) .
\end{aligned}
$$

The optimum sequence $s_{0}^{(0)}, s_{1}^{(0)}, s_{1}^{(1)}, s_{2}^{(1)}, \ldots$ converges to the sum

$$
0.761310204001103486
$$

as quickly as in the other cases. In particular, $s_{8}^{(8)}$ has 14 correct decimal digits.

\section{ACKNOWLEDGMENT}

We wish to express our gratitude to the referees for their helpful comments and suggestions which have improved the original manuscript.

\section{BIBLIOGRAPHY}

1. M. Abramowitz and I. A. Stegun (eds.), Handbook of mathematical functions, Nat. Bureau of Standards, Washington, 1964.

2. W. N. Bailey, Contiguous hypergeometric functions of the type ${ }_{3} F_{2}(1)$, Proc. Glasgow Math. Assoc. 2 (1954), 62-65.

3. C. Brezinski and M. Redivo Zaglia, Extrapolation methods. Theory and practice, NorthHolland, Amsterdam, 1991.

4. A. Erdélyi (ed.), Higher transcendental functions, McGraw-Hill, New York, 1953.

5. I. S. Gradshteyn and I. M. Ryzhik, Tables of integrals, series and products, Academic Press, New York, 1980.

6. S. L. Kalla, S. Conde, and Y. L. Luke, Integrals of Jacobi functions, Math. Comp. 38 (1982), 207-214.

7. K. Knopp, Theory and application of infinite series, Hafner, New York, 1949.

8. Y. L. Luke, The special functions and their approximations, Academic Press, New York, 1969.

9.

10. _ Rational approximations for the logarithmic derivative of the gamma function, Appl. Anal. 1 (1979), 65-73. 
11. A. Sidi, Convergence properties of some nonlinear sequence transformations, Math. Comp. 33 (1979), 315-326.

12. _ Analysis of convergence of the T-transformation for power series, Math. Comp. 35 (1980), 833-850.

13. E. J. Weniger, Nonlinear sequence transformations for the acceleration of convergence and the summation of divergent series, Comp. Phys. Reports 10 (1989), 189-371.

14. J. Wimp, Irreducible recurrences and representation theorems for ${ }_{3} F_{2}(1)$, Comput. Math. Appl. 9 (1983), 663-678.

15. __ Computation with recurrence relations, Pitman, Boston-London, 1984.

16. D. Zeilberger, Gauss's ${ }_{2} F_{1}(1)$ cannot be generalized to ${ }_{2} F_{1}(x)$, J. Comput. Appl. Math. 39 (1992), 379-382.

Institute of Computer Science, University of Wroclaw, ul. Przesmyckiego 20, 51-151 Wroclaw, Poland

E-mail address: sle@ii.uni.wroc.pl

Institute for Low Temperature and Structure Research, Polish ACADEmy of ScIences, P.O. BoX 937, 50-950 Wroclaw, PolaND

E-mail address: intibs@plwrtu11.bitnet 